\title{
Ion Formation Processes in the Afterpeak Time Regime of Pulsed Glow Discharge Plasmas
}

\author{
Changkang Pan and Fred L. King \\ Department of Chemistry, West Virginia University, Morgantown, West Virginia, USA
}

\begin{abstract}
The formation of ions following the termination of power in a pulsed glow discharge ion source is investigated. The populations of ionized species containing sputtered atoms $\mathbf{M}^{+}$, $\mathrm{M}_{2}^{+}$, and $\mathrm{MAr}^{+}$are observed to maximize after the termination of discharge power. Collisions involving sputtered atoms and metastable argon atoms, Penning and associative ionization, are considered to be responsible for the formation of ions in the discharge afterpeak time regime. The domination of these ion formation processes during the afterpeak time regime is supported by the results from investigations of discharge operating parameters, metastable argon atom quenching, and ion kinetic energy distributions. (/ Am Soc Mass Spectrom 1993, 4, 727-732)
\end{abstract}

$\mathrm{G}$ low discharge mass spectrometry (GD/MS) is often the method of choice for the direct determination of trace elements in solid-state materials [1]. Analytical GD/MS commonly uses a steady-state argon GD for the atomization and ionization of analyte species. Recent reports indicate that pulsed GDs can uffer certain andytical advantages [2-4]. Among these advantages is the ability to differentiate discharge gas ions from analyte ions through time-resolved mass spectrometry measurements.

Sample atomization in GD ion sources is the result of cathodic sputtering [1]. In cathodic sputtering, the discharge operating current and voltage respectively control the rate and energy of discharge gas atom bombardment and, thereby, control the rate of sample atomization. Operating pressure influences sputter atomization principally through its impact on the relationship between current and voltage. As the discharge pressure increases, either the operating current increases to maintain a constant voltage or the operating voltage decreases to maintain a constant current. It should be kept in mind that the product of current and voltage, power, controls the atomization. Although higher operating powers yield enhancements in both atomization and sensitivity, discharge instability and sample overheating limit the maximum operating power in steady-state analytical GDs. One approach to obtain the advantages of higher power operation, without suffering the seemingly concomitant disadvantages of discharge instability and sample overheating, is the operation of analytical GDs in a pulsed-power

Address reprint requests to Fred L. King, Department of Chemistry, West Virginia University, Box 6045, Morgantown, WV 26506-6045. modc. Such operation yiclds greater average ion signal intensities than DC operation at the same average operating current [4].

Harrison and co-workers $[4,5]$ report that the operation of GD ion sources at frequencies of up to $100 \mathrm{~Hz}$ results in pronounced differences in the temporal profiles of discharge gas ions and andlyte ions. Three distinct time regimes exist during each discharge on/off cycle. The discharge gas ion signal maximizes immediately following the application of discharge power, the prepeak regime. The discharge gas ion signal then decays to and remains at a constant value, the plateau regime, until discharge power is terminated. On the termination of discharge power, the discharge gas ion signal disappears. The analyte ion signal exhibits markedly different behavior. The analyte ion signal gradually increases to reach a steady state, the plateau regime, at approximately the same time as the discharge gas ion signal. On removal of discharge power, the analyte ion signal rapidly increases to a maximum, the afterpeak regime, after the decay of the discharge gas ion signal. Significantly, this afterpeak affords the analyst the ability to observe ionized sputtered atoms in the virtual absence of discharge gas ions.

The results from mass spectrometry investigations of the afterpeak ionization process are reported here. The occurrence of a unique ionization process during the afterpeak time regime is indicated by the existence of atomic and polyatomic ions containing sputtered atoms in the absence of discharge gas ions. Information about the identity of the afterpeak ionization process is obtained from investigations of the influence of discharge operating pressure, discharge operating power, discharge sampling region, and discharge gas compo- 
sition on the ion signal temporal profile. The ion kinetic energy distributions for plateau and afterpeak regions are presented for ions arising from sputtered atoms.

\section{Experimental}

\section{Glow Discharge Ion Source}

The GD ion source used in these investigations is housed in a six-way stainless steel cross, fitted with $6.985-\mathrm{cm}$ conflat flanges. The sample cathode is introduced by means of a direct insertion probe through a $1.27-\mathrm{cm}$ ball valve mounted on the cross. The mass spectrometer sampling port, $0.5 \mathrm{~mm}$ in diameter, is mounted directly opposite to the probe inlet. The GD is operated with reagent-grade argon at pressures ranging from 0.5 to 1.0 torr, as measured by a thermocouple gauge (Teledyne Hastings DV-4, Hampton, VA) at the gas inlet port. Sample cathode disks, $5.0 \mathrm{~mm}$ in diameter and $4.5 \mathrm{~mm}$ in height, are formed from bulk materials by machining or from powdered material by compaction. The discharge is powered by a fast-response operational power supply (Kepco OPS-3500, Flushing, NY). Pulsing is achieved by driving this power supply with the step function output from a pulse generator. The output voltage of the power supply is delayed by a $0.7-\mathrm{ms}$ delay, with respect to the input pulse.

\section{Mass Spectrometry System}

A triple quadrupole mass spectrometer (Extrel ELQ400, Export, PA), operating with the third quadrupole as the mass analyzer, was used in these investigations. This instrument was adapted to permit investigation of GD ion sources (Figure 1). The standard chemical ionization/electron ionization ion source was replaced with a Bessel-box kinetic energy analyzer. Ions were focused from the sampling port into the entrance of the energy analyzer by means of an Einzel lens assembly. Sequential stages of differential pumping were used between the GD chamber (0.5-1.0 torr), the energy analyzer region $\left(10^{-5}\right.$ torr), and the mass analyzer $\left(10^{-6}\right.$ torr) to permit mass spectrometry sampling of the GD plasma. Temporal ion signal profiles were monitored with the third quadrupole set to transmit a specific mass-to-charge ratio ion. These profiles were acquired with a digital storage oscilloscope (Tektronix 2232, Beaverton, OR) connected through a preamplifier (MIT Combo 100, Boulder, CO) to the output of the continuous dynode electron multiplier.

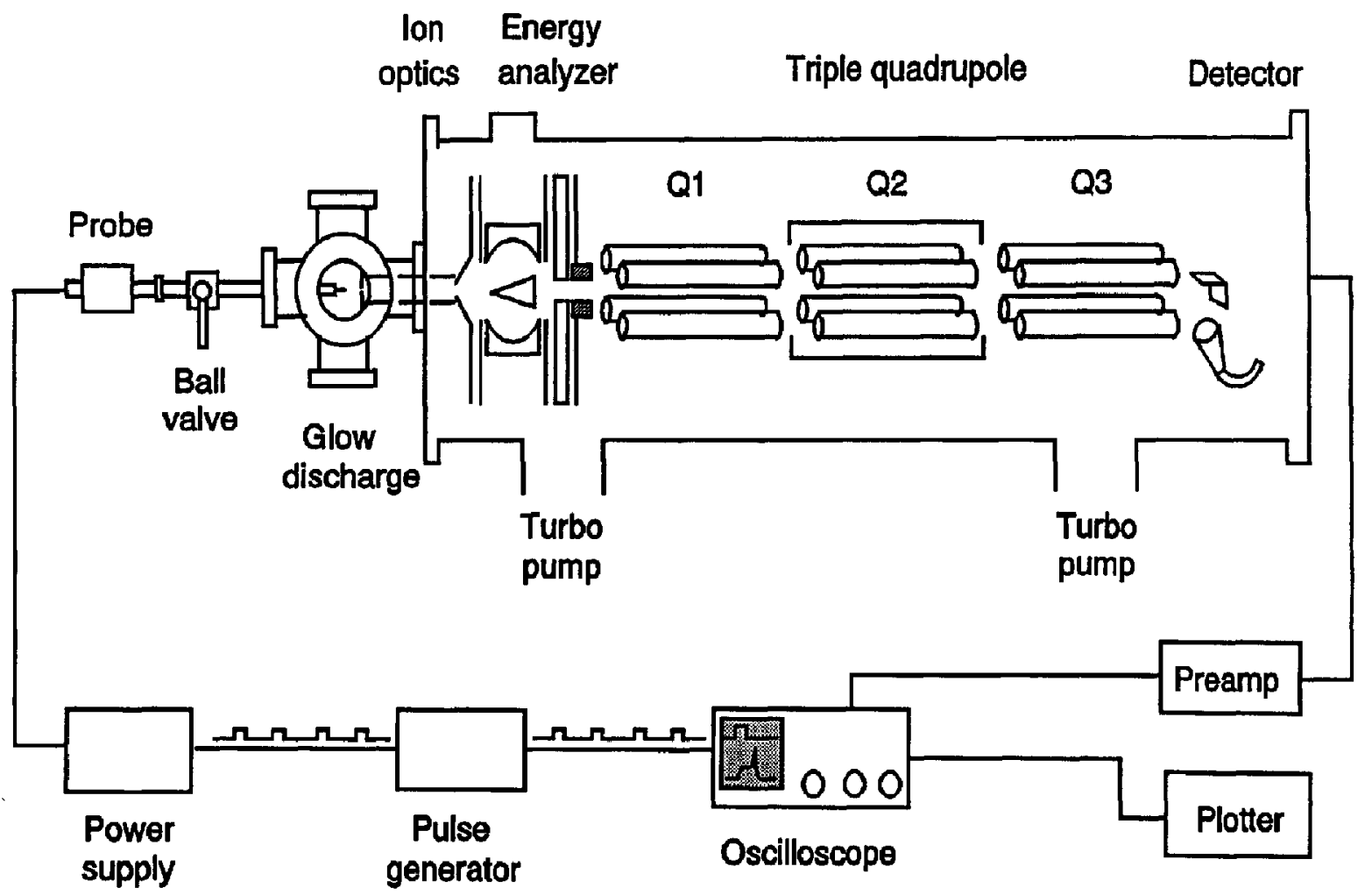

Figure 1. Experimental arrangement of pulsed GD/MS system. 


\section{Results and Discussion}

\section{Temporal Profiles of Ions Containing Sputtered Atoms}

Operation of the GD with the output power pulsed at $50 \mathrm{~Hz}$ and $25 \%$ duty cycle yields the signal profile for ${ }^{107} \mathrm{Ag}^{+}$shown in Figure 2a. As reported by other groups $[4,5]$, the ionized sputtered atoms exhibit temporal profiles in which there is an initial region of steady signal increase, the prepeak, followed by a temporal regime of stable signal, the plateau, and ending with a surge in ion signal, the afterpeak. The steady increase in ionized sputtered atom signal during the prepeak regime results from the initiation of sputtering following the application of discharge power. There is an induction period during which the discharge support gas undergoes electrical breakdown, and the first argon ions form [6]. Once formed, these ions bombard the sample surface and initiate sputtering. The sputtered atom population increases to a constant level after the initiation of sputtering. The steady-state ion signal observed in the plateau region is analogous to that obtained in continuous DC operation. During this time period, the plasma is in a quasi-equilibrium state in which rates of atomization and ionization are constant [6]. On termination of the applied discharge power, the sputtered ion signal rapidly increases to a maximum and then decays. Temporal signal profiles for the

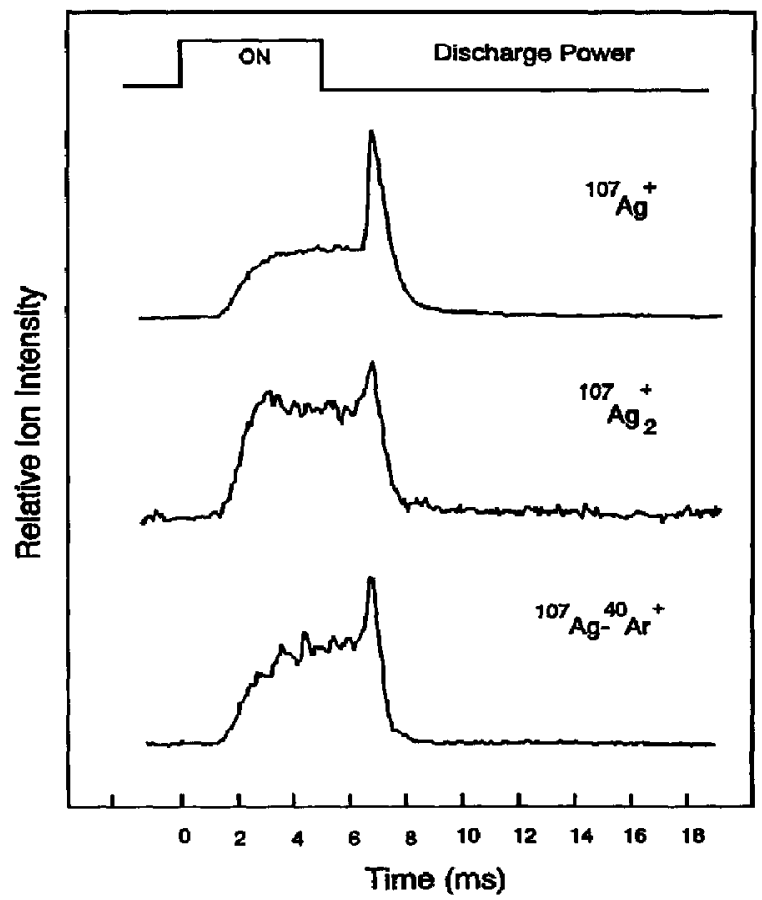

Figure 2. Temporal signal profiles of ${ }^{107} \mathrm{Ag}^{+},{ }^{107} \mathrm{Ag}_{2}^{+}$, and ${ }^{10} \mathrm{Ag}^{40} \mathrm{Ar}^{+}$; power $1.1 \mathrm{~W}$, pressure 0.7 torr, and sampling distance $10 \mathrm{~mm}$. diatomic species ${ }^{107} \mathrm{Ag}_{2}^{+}$and ${ }^{107} \mathrm{Ag}^{40} \mathrm{Ar}^{+}$are shown in Figures $2 b$ and $c$, respectively. Both species exhibit profiles similar to that of the silver atomic ion, indicating that they arise from processes involving a common precursor. The precursor common to the formation of all of these species appears to be the metastable argon atom. The processes involving this species and leading to the formation of the ions shown in Figure 2 are shown in eq 1-3:

Penning ionization,

$$
\begin{aligned}
\mathrm{Ar}^{* \mathrm{~m}}+\mathrm{Ag}_{2} & \rightarrow \mathrm{Ag}_{2}^{+}+\mathrm{Ar}+e^{-} \\
\mathrm{Ar}^{* \mathrm{~m}}+\mathrm{Ag} & \rightarrow \mathrm{Ag}^{+}+\mathrm{Ar}+e^{-}
\end{aligned}
$$

Associative ionization,

$$
\mathrm{Ar}^{* \mathrm{~m}}+\mathrm{Ag} \rightarrow \mathrm{ArAg}^{+}+e^{-}
$$

An examination of the origin of metastable argon atoms provides evidence that these species would be expected to dominate the afterpeak time regime. Metastable argon atoms form as a result of the recombination of argon ions with thermalized electrons [7, 8]. The recombination yields an argon atom in an excited $5 p$ level, Ar*. This excited atom decays radiatively to a metastable $4 \mathrm{~s}$ level, $\mathrm{Ar}^{* \mathrm{~m}}$. Such recombination is the dominant process of discharge decay following the termination of applied power. This leads to an increase in the population of long-lived metastable argon atoms immediately following discharge power termination. Likewise, processes involving these atoms, as in eq 1-3, dominate ion production during the time regime following discharge power termination. Because the influence of key operating parameters on the population of metastable argon atoms is known from previous studies [9, 10], changes in the temporal profile of the ionized atoms as a function of operating conditions are examined for correlation with predictable changes in metastable argon atom populations. The remaining discussion focuses on the results of such studies.

\section{Influence of Discharge Pressure}

The temporal profile of ${ }^{63} \mathrm{Cu}^{+}$was monitored as the discharge pressure was increased over a range from 0.55 to 1.0 torr (Figure 3). At the lowest pressure ( 0.55 torr, Figure $3 a$ ), the ion signal exhibits a small afterpeak. As the pressure was increased (Figure $3 \mathrm{~b}-\mathrm{d}$ ), the afterpeak-to-plateau ion signal ratio increased to a steady value, whereas the overall signal decayed. During these changes in pressure, the discharge current was held constant, and thus the discharge voltage decreased as the pressure increased. Previous studies have shown that the population of metastable argon atoms has a bell-shaped response to increasing pressure under constant current conditions $[9,10]$. As the discharge pressure is decreased, discharge voltages and electron kinetic energies are increased to sustain the GD. Because metastable argon 


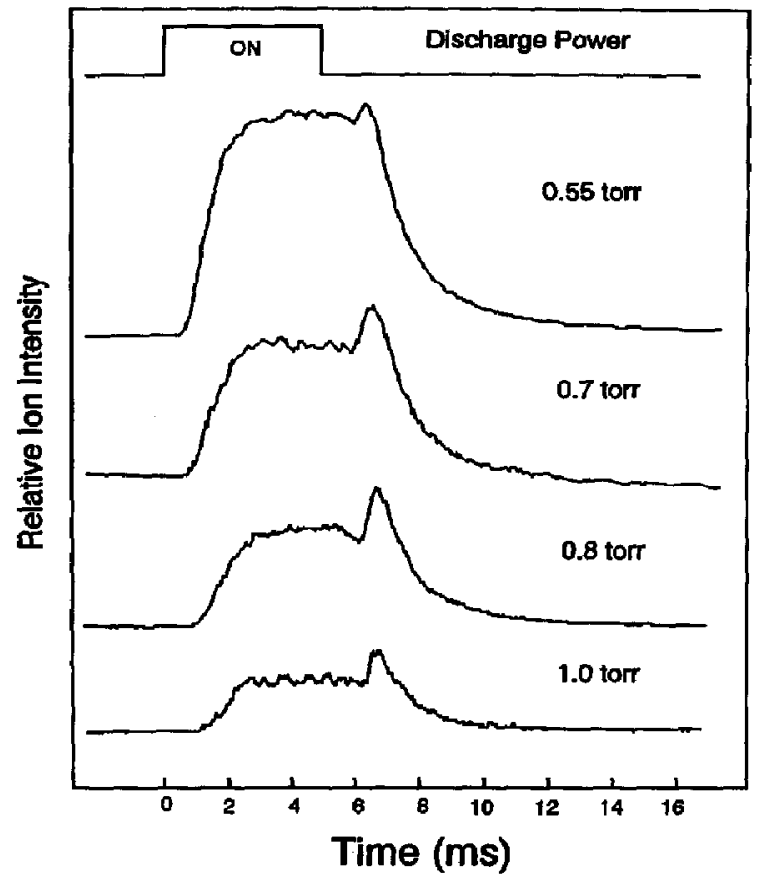

Figure 3. Temporal profiles of ${ }^{63} \mathrm{Cu}^{+}$at pressures of $0.55,0.7$, 0.8 , and 1.0 torr; constant power $1.1 \mathrm{~W}$ and constant sampling distance $10 \mathrm{~mm}$.

atoms form via recombination of electrons with argon ions, and the cross section for this recombination increases with decreasing electron kinetic energy, their population will increase as electron kinetic energies decrease with increasing pressure. At higher pressures a competitive process, collisional deexcitation, begins to reduce their population. Further increases in pressure result in a steady decrease in the population of metastable argon atoms as a result of increases in the probability of deexcitation through collisions [11]. The intensity of analyte ion signals in the afterpcaks rela tive to those in the plateaus, as shown in Figure 3, follows the trend in metastable argon atom populations as a function of discharge pressure. The general decrease in overall signal arises because of the decrease in sputtered atom population arising from the decrease in discharge operating voltage. The approach to a constant afterpeak-to-plateau signal ratio would be expected because of the increased contribution of Penning ionization in the plateau as the pressure increases. It is known that up to $80 \%$ of sputtered atom ionization in conventional DC-powered GD/MS sources results from Penning mechanisms involving metastable argon atoms [12]. A possible alternative explanation for the afterpeak is that it arises from charge exchange between argon ions and metal atoms; however, under conditions of constant current, increasing pressure, and decreasing applied potential, the cross section for such charge-exchange ionization should be increasing, not decreasing, as observed in these studies.

\section{Influence of Discharge Power}

A series of measurements were made under conditions of constant discharge pressure but increasing voltage and current. In this study, the applied discharge power was monitored because voltage and current could not be controlled independently. Increasing the power from 1.1 to $7.8 \mathrm{~W}$ was observed to increase the signal intensity and the afterpeak-to-plateau ratio. In the pressure studies discussed previously, it was noted that metastable argon atom populations decreased as the applied voltage increased; however, in those studies the current was maintained at a constant value. In these power studies the current was increasing along with the voltage; thus, more argon ions were available for recombination, resulting in a net increase in the relative probability of Penning ionization. Similar trends with increased power were observed previously for conventional DC-powered GDs [12].

An additional change observed in these profiles resulting from the increase in power was a shift in the temporal position of the afterpeak maximum to increasingly longer times with respect to the termination of discharge power (Figure 4). This temporal shift results from the increase in electron kinetic energies.

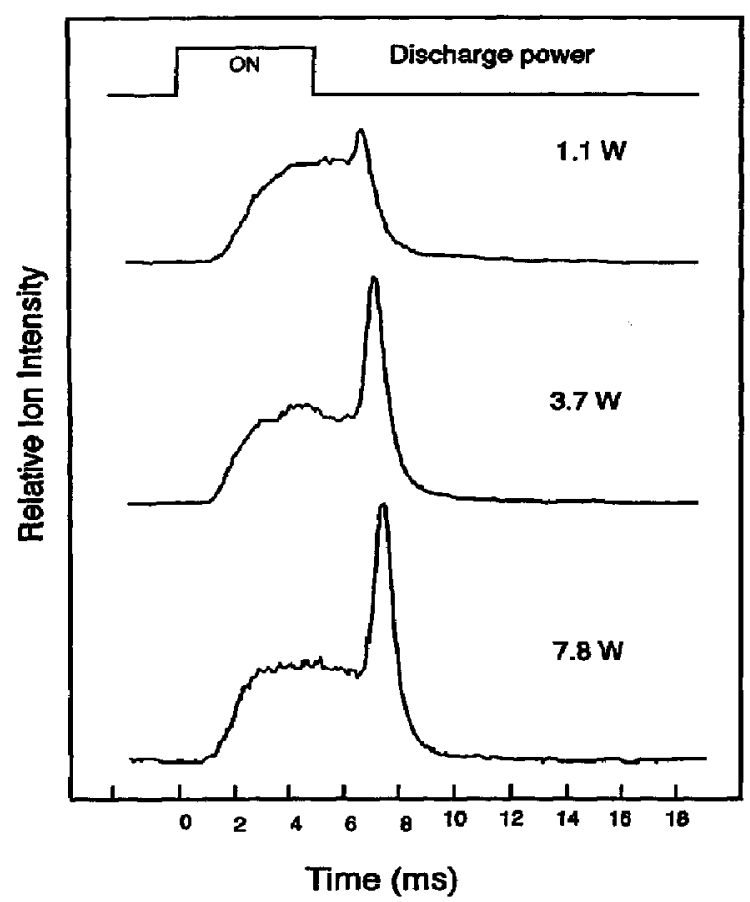

Figure 4. Temporal profiles of ${ }^{63} \mathrm{Cu}^{+}$at powers of 1.1, 3.7, and $7.8 \mathrm{~W}$; constant pressure 0.7 torr and constant sampling distance $10 \mathrm{~mm}$. 
The time necessary for the electrons to thermalize and to maximize their cross section for recombination with argon ions increases as the electron kinetic energy increases [6]. The result of this increased thermalization time is a shift in the metastable argon atom population maximum. The shift in the afterpeak strongly indicates that the formation of afterpeak ions results from processes relying on metastable argon atoms.

\section{Influence of Plasma Sampling Region}

The mass spectrometer samples ions formed in the near vicinity of the ion exit orifice. Movement of the sample cathode away from this ion exit orifice effectively changes the region of the plasma sampled by the mass spectrometer. One significant effect of sampling farther away from the cathode is the increase in the distance that sputtered atoms must travel prior to their ionization. Figure 5 shows how the ${ }^{63} \mathrm{Cu}^{+}$temporal signal profile changes as the cathode is moved from 6 to $25 \mathrm{~mm}$ away from the ion exit orifice. As this separation distance increases, the afterpeak signal decreases relative to the plateau signal, and the onset of the plateau shifts farther in time from the initiation of the discharge power pulse. These effects are the result of decreases in sputtered atom and metastable argon atom concentrations at increasing distances from

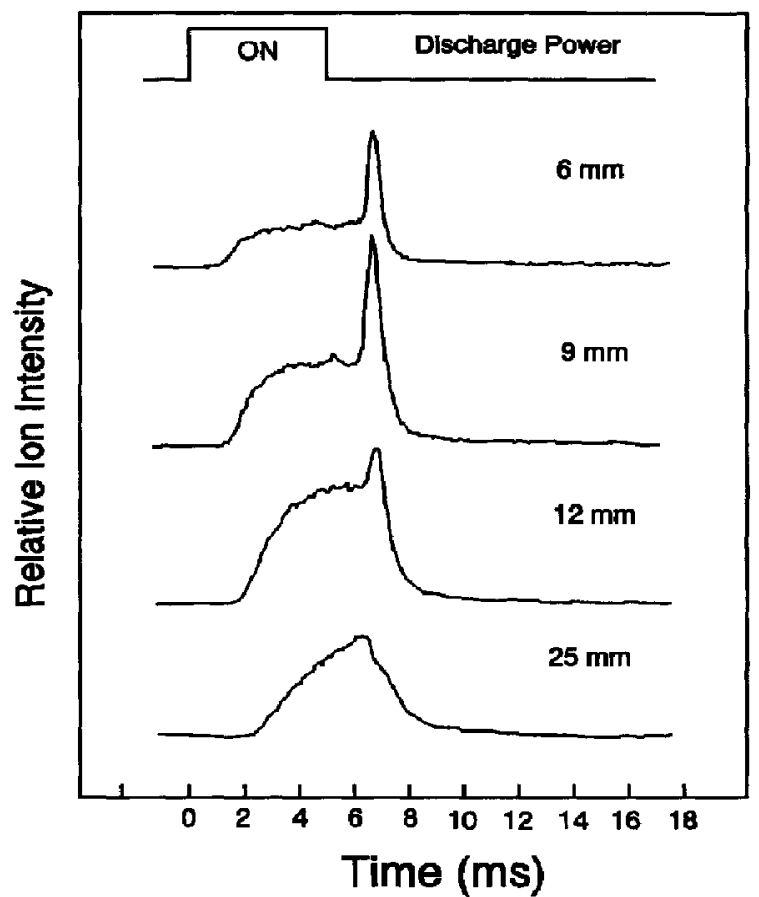

Figure 5. Temporal profiles of ${ }^{63} \mathrm{Cu}^{+}$sampled with cathode/ion exit orifice separation distances of $6,9,12$, and $25 \mathrm{~mm}$; constant power $1.1 \mathrm{~W}$ and constant pressure 0.7 torr. the cathode. Both sputtered atom and argon ion densities maximize in the region closest to the cathode surface. At greater distances from the cathode, both argon ion and sputtered atom densities decrease. The decrease in the afterpeak signal relates to the decrease in argon ion densities. If the afterpeak arises from Perning ionization involving collisions between metastable argon atoms and sputtered atoms, and the only source of metastable argon atoms is the recombination of electrons with argon ions, then the afterpeak intensity should decline as the argon ion population decreases. The temporal shift in the onset of the plateau arises from the time required for cathode atoms to be sputtered and to diffuse to the sample region.

\section{Influence of Metastable Quenching}

Hess and co-workers $[9,10,12]$ demonstrated that the addition of a metastable argon atom quenching reagent, such as methane, reduces the contribution of metastable argon atom-mediated processes, such as Penning ionization, to sputtered-atom excitation and ionization in GD plasmas. Methane lowers the population of metastable argon atoms not only by collisional deexcitation of these species (quenching), but also by reduction of the precursor argon ion population through charge exchange. Charge exchange also influences the atomization of the sample cathode. The conversion of argon ions to methyl fragment ions lowers the rate of atomization because the lighter methyl fragment ions are less effective in sputtering the analyte callode.

Methane addition to the argon at levels of $5 \%$ and $10 \%$ of the total pressure results in the temporal profile changes portrayed in Figure 6. The addition of methane at the $5 \%$ level effectively eliminates the afterpeak signal contribution but decreases the plateau signal by only $25 \%$. This result is consistent with the quenching of metastable argon atoms that form during the afterpeak but cannot be replenished. The plateau

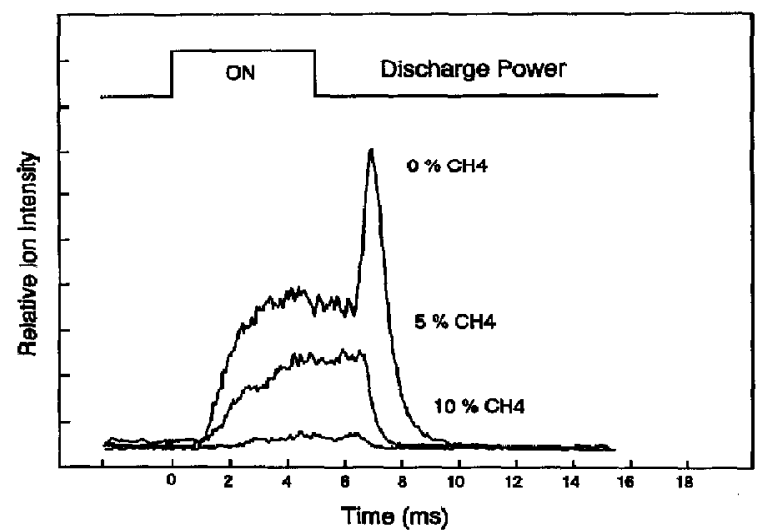

Figure 6. Effect of the addition of methane at $0 \%, 5 \%$, and $10 \%$ on the temporal profile of the ${ }^{6.3} \mathrm{Cu}^{+}$species. 
ion signal is affected to a lesser extent, indicating that analyte atoms are sputtered and are subject to ionization by steady-state processes. The addition of methane at the $10 \%$ level effectively eliminates the plateau signal as well. This is consistent with decreases in both steady-state metastable argon atom and sputtered-atom populations.

\section{Ion Kinetic Energy Distributions}

The Bessel-box energy analyzer was used to evaluate the relative kinetic energy distributions of ions generated during the plateau and afterpeak regions of the temporal profile. In Figure 7 the relative signal is plotted as a function of the Bessel-box ion energy setting. As seen in Figure 7 , the ions generated in the afterpeak exhibit greater kinetic energies but with a narrower distribution. The increase in ion kinetic energy is a result of the collapse of the plasma on the termination of applied power. During the steady state, the plasma is charge neutral, with the positive ions effectively shielded by the more mobile electrons [6]. On termination of the plasma, the electron population is subject to depletion through collisions with chamber walls at a rate greater than that expected for the loss of ions because of the greater relative mobility of electrons. Such depletion should result in a positive increase in net plasma potential. A positive increase in the net plasma potential would increase the kinetic energy with which ions leave the plasma, hence the observed shift to slightly higher ion kinetic energies. The narrowing of the kinetic energy distribution for afterpeak ions is consistent with the domination of ionization during the afterpeak time regime by a single

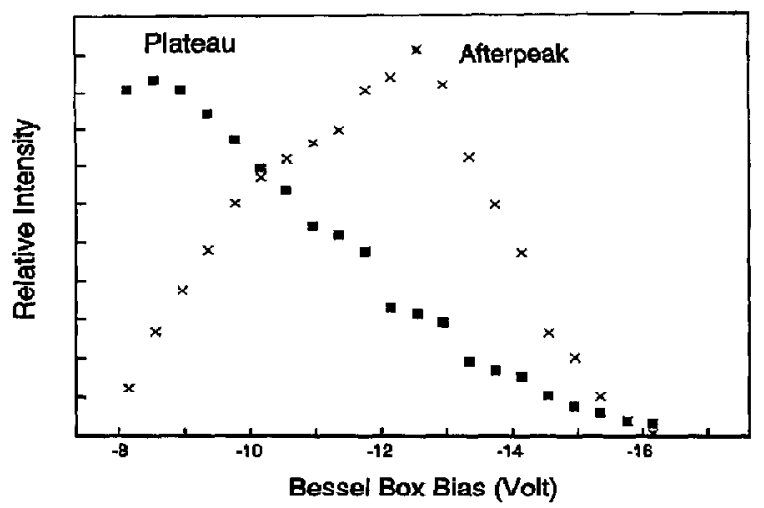

Figure 7. Relative ion kinetic energy distribution for ${ }^{63} \mathrm{Cu}^{+}$ during the plateau and afterpeak time regimes; power $1.1 \mathrm{~W}$, pressure 0.7 torr, and sampling distance $10 \mathrm{~mm}$. process. Ionization in the plateau results from a combination of processes, such as electron collisions, charge-exchange collisions, and argon metastable atom collisions. The observed kinetic energy distribution arises from the combination of the kinetic energy distributions from each of these individual ionization processes. During the afterpeak, only Penning processes appear to contribute to analyte ionization.

\section{Conclusions}

The temporal profiles of ions generated in pulsed GDs were examined. Ions containing sputtered atoms $\mathrm{M}^{+}$, $\mathrm{M}_{2}^{+}$, and $\mathrm{MAr}^{+}$exhibit similar temporal profiles in which ion signal maximizes sharply following the termination of the applied discharge power. The effects of operating parameters, such as pressure, power, and plasma sampling, indicate that the afterpeak signal results from enhanced ionization through collisions with metastable argon atoms. The metastable argon atoms that participate in this ionization arise from recombination of argon ions with electrons during the time following discharge power termination. All species that exhibit afterpeaks form by processes involving these metastable argon atoms and sputtered atoms. The narrowing of the kinetic energy distribution of afterpeak ions in comparison to plateau ions indicates that the afterpeak ions arise from collisions with metastable argon atoms.

\section{References}

1. King, F. L.; Harrison, W. W. Mass Spectrom. Rev. 1990, 9, 285-317.

2. Glick, M.; Snith, B. W.; Winefordner, J. D. Aral. Chem. 1990, 62, 157-161.

3. Chakrabarti, C. L.; Headrick, K. L.; Hutton, J. C.; Bicheng, Z.; Bertels, P. C.; Back, M. H. Anal. Chem. 1990, 62, 574-586.

4. Klingler, J. A-; Savickas, P. J.; Harrison, W. W. Anal. Chem. 1990, 1, 138-143.

5. Klingler, J. A.; Barshick, C. M.; I Iartison, W. W. Anal. Chem. 1991, 63, 2571-2576.

6. Chapman, B. Glow Discharge Processes; Wiley: New York, 1980.

7. Biondi, M. A. Phys. Rev. 1963, 129, 1181-1188.

8. Strauss, J. A.; Ferreira, N. P.; Human, H. G. C. Spectrochim. Acta 1982, 37B, 947-954.

9. Smith, R. L.; Serxner, L. D.; Hess, K. R. Anal. Chem. 1989, 61, $1103-1108$.

10. Serxner, L. D.; Smith, R. L.; Hess, K. R. Appl. Spectrosc. 1991, $45,1656-1664$.

11. Biondi, M. A. Phys. Rev. 1952, 88, 660-665.

12. Levy, M. K.; Serxner, D.; Angstadt, A. D.; Smith, R. L.; Hess, K. R. Spectrochim. Acta 1991, 46B, 253-267. 